\title{
The Green's Function Method for the Supported Plate Boundary Value Problem
}

\author{
S. H. SCHOT
}

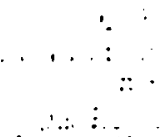

The deflection $u$ of a thin elastic plate is governed by the biharmonic equation $\Delta^{2} u=0$, where $\Delta$ is the two-dimensional Laplace operator. The problem of solving this equation in the domain $D$ occupied by the plate when $u$ and $\Delta u$ are assigned on the boundary $\partial D$ is of ten called the supported plate boundary value problem. Strictly speaking this terminology is not correct since $\Delta u$ should bo replaced by the more complicated expression for the plate's moment $M(u)$ on $\partial D$; however, when $\partial D$ consists only of rectilinear segments (or when the Poisson ratio is unity) $M(u)$ reduces to $\Delta u$. Hore, the supported plate problem is solved by a Green's function method, closed form solutions are obtained for the disk and tho half-plane, and the supported plate Green's functions for these domains are computed explicitly. As a chock, the solutions of these boundary value problems are also derived using a modification of the GoursatAlmansi method.

Key words: Biharmonic equation, supported elastic plate, boundary value problems, Green's function

AMS subject classification: $31 \mathrm{~A} 30,35 \mathrm{~J} 40$

\section{Introduction}

The bending of a thin elastic plate, initially occupying a bounded domain $D$ in the $(x, y)$-plane, by a force perpendicular to the plate is governed by the equation

$$
\Delta^{2} u=F(x, y)
$$

in $D$, where $\Delta^{2}=\left(\partial^{2} / \partial x^{2}+\partial^{2} / \partial y^{2}\right)^{2}$ is the biharmonic operator, $u$ is the normal deflection of the plate, and $F(x, y)$ is the normal force per unit area divided by the flexural rigidity of the plate. One of the following three types of boundary conditions is usually imposed at the edge $\partial D$ of the plate.
(a) clamped plate
$u=0, \quad \partial u / \partial n=0$
(b) supported plate
$u=0, \quad \Delta u=0$
(c) free plate
$\Delta u=0, \partial \Delta u / \partial n=0$.

Here $n$ represents the exterior normal to the boundary $\partial D$ of $D$. Strictly speaking, the nomenclature used for (b) and (c) is not correct, for these boundary conditions should be formulated in the more general form

(b) $u=0, \quad M(u) \equiv \Delta u-(1-x)\left(u_{o \sigma}-K u_{n}\right)=0$

(c) $M(u)=0, \quad N(u)=(\Delta u)_{n}+(1-x)\left(u_{o n}-K u_{o}\right)_{0}=0$,

where $K$ and $\sigma$ are the curvature of $\partial D$ and the arclength along $\partial D$, respectively, and $x$ is the Poisson ratio [15]. However, the terminology stated above seems to have become accepted in 
the mathematical literature [7]. When the boundary $\partial D$ consists of rectilinear segments (as is the case for the half-plane in Section 5 below) it follows that $K=0$ and $u_{o o}=0$ along these segments and hence the boundary condition $u=\Delta u=0$ on $\partial D$ is correct for all $x$.

Closely related to the above problem is the boundary value problem in which a plate free from normal load is subjected to displacements and slopes along its edge. In this case the deflection of the plate is governed by the biharmonic equation

$$
\Delta^{2} u=0
$$

in $D$, subject to one of the following three types of boundary conditions on $\partial D$ :

$$
\begin{aligned}
& \text { (a') } u=f_{1}, \quad \partial u / \partial n=f_{2} \\
& \text { (b') } u=g_{1}, \quad . \quad . \Delta u=g_{2} \\
& \text { (c) } \Delta u=h_{1}, \quad \partial \Delta u / \partial n=h_{2}
\end{aligned}
$$

where $f_{1}, f_{2}, \ldots, h_{2}$ are continuous functions assigned on $\partial D$. By analogy with problems $(1) /(\mathrm{a})$, (1) $/$ (b) and (1) $/\left(\right.$ c) above, the three problems $(2) /\left(a^{\prime}\right),(2) /\left(b^{\prime}\right)$ and $(2) /\left(c^{\prime}\right)$ are often called the clamped plate, supported plate, and free plate homogeneous boundary value problems, respectively. These problems have also been termed the first, second, and third boundary value problem for the biharmonic equation $[2,6]$.

Of these three boundary value problems, only $(2) /\left(a^{\circ}\right)$ has received much attention in the literature. The classical Green's function method of potential theory has been extended to this problem, and closed form expressions for clamped plate Green's functions have been obtained for the disk, half-plane, limacon, and other regions $[3,9,11]$. Moreover, a direct method for solving problem $(2) /\left(\mathrm{a}^{\prime}\right)$ was developed by Almansi [1] and others $[10,14]$. This method obviates the calculation of Green's functions and uses forms of Goursat's formula [8] to reduce $(2) /\left(a^{\prime}\right)$ to two Dirichlet problems for the given domain. When the domain is a disk, Goursat's formula asserts that any biharmonic function $u$ defined in a disk $|x|<R$ (i.e. $u \in C^{4}$ and $\Delta^{2} u=$ 0 in $|x|<R)$ can be represented by

$$
u=|x|^{2} \Phi+\Psi
$$

where $\Phi$ and $\Psi$ are uniquely determined harmonic functions in $|x|<R$. Conversely, the formula (3) in which $\Phi$ and $\Psi$ are harmonic in $|x|<R$ represents a biharmonic function $u$ in $|x|<R$.

The supported plate boundary value problem $(2) /\left(b^{\circ}\right)$ seems to have been largely ignored, and it is surprising that the supported plate Green's functions for such common domains as the disk and the half-plane have not even been computed explicitly. However, Reissner [13] obtained the Green's function for a disk in the closely related case of an elastically clamped plate.

In this paper, a Green's function method is developed for problem $(2) /\left(b^{\prime}\right)$ which yields the solution of this problem in terms of the supported plate Green's function and the stretched membrane (harmonic) Green's function for the given domain. The supported plate Green's functions are computed explicitly for the disk and the half-plane by a complex variable method first suggested for the clamped plate by Garabedian [5,6]. Using these Green's functions, explicit integral formulas for the solutions of the homogeneous supported plate boundary value problems are then obtained for these domains. The solutions are also derived without the use of Green's functions by extending Almansi's direct method to these supported plate problems. 


\section{The Green's function method}

The Green's function method for the supported plate utilizes the Green's identities which have been used to solve the clamped plate boundary value problem. Hence they will first be reviewed here briefly $[4,6]$.

Let $D$ be a simply-connected plane domain bounded by a simple smooth curve $\partial D$. Let $u$ and $v$ possess continuous partial derivatives up to and including order four on the closed domain $\bar{D}=D+\partial D$. Then Green's second identity for the biharmonic operator is

$$
\iint_{D}\left(u \Delta^{2} v-v \Delta^{2} u\right) d A=\iint_{\partial D}\left(\Delta u \frac{\partial v}{\partial n}-\Delta v \frac{\partial u}{\partial n}+u \frac{\partial \Delta v}{\partial n}-v \frac{\partial \Delta u}{\partial n}\right)_{\cdots} d \sigma, \ldots
$$

where $n$ is the exterior normal to the boundary $\partial D$, do is the element of arclength along $\partial D$, and $d A$ is the area element of $D$. Now introduce the fundamental solution $S(r)=r^{2} \log r$ of the biharmonic equation (2), where $r$ is the distance between the points $P \in D$ and $Q \in \bar{D}$. If $P$ is surrounded by a disk $D_{\varepsilon}(P) \subset D$ of radius $\varepsilon$, the identity (4) with $v=S(r)$ is applied to $D$ $D_{\varepsilon}(P)$, and the limit as $\varepsilon \rightarrow 0$ is taken, then there results Green's third identity for the biharmonic operator, namely

$$
u(P)=\iint_{\partial D}\left(\Delta u \frac{\partial S}{\partial n}-\Delta S \frac{\partial u}{\partial n}+u \frac{\partial \Delta S}{\partial n}-S \frac{\partial \Delta u}{\partial n}\right) d \sigma+\iint_{D} S \Delta^{2} u d A .
$$

If this identity is to be used to solve (1)/(b'), the terms in the boundary integral of (5) which contain normal derivatives of $u$ and $\Delta u$ must now be eliminated. This may be accomplished by introducing the supported plate Green's function [7]

$$
\Gamma(Q, P)=S(r)+\gamma(Q, P),
$$

where $\gamma \in C^{4}$ in $\bar{D}$, satisfies $\Delta^{2} \gamma=0$ in $D$, and takes on the boundary values $\gamma=-S$ and $\Delta \gamma=$ $-\triangle S$ on $\partial D$. This definition implies that $\Delta^{2} \Gamma=0$ for $Q \neq P, Q \in D$; asymptotically $\Gamma \sim S$ for $Q=$ $P$; and $\Gamma=0=\Delta \Gamma$ for $Q \in \partial D$. Letting $v=\gamma(Q, P)$ in (4), adding the result to (5), and applying the boundary conditions of $\Gamma$ on $\partial D$, there results

$$
u(P)=\iint_{\partial D}\left(\Delta u \frac{\partial \Gamma}{\partial n}+u \frac{\partial \Delta \Gamma}{\partial n}\right) d \sigma+\iint_{D} \Gamma \Delta^{2} u d A
$$

This identity may be simplified further by noting an important property of the supported plate Green's function which does not hold for the clamped plate Green's function [7]: Let the harmonic Green's function be denoted by $G(Q, P)$, i.e. the function which has the properties $\Delta G=0$ for $Q \neq P, Q \in D$; asymptotically $G \sim T(r)$ в $(1 / 2 \pi) \log (1 / r)$ for $Q=P$; and $G=0$ for $Q \in$ $\partial D$. Then it follows from the definitions of $\Gamma$ and $G$ and from the relation $\Delta S=-T$ that

$$
\Delta \Gamma(Q, P)=-G(Q, P), Q \neq P .
$$

When ( 8 ) is substituted into (7) the latter expression becomes

$$
u(P)=\int_{\partial D}\left(\Delta u \frac{\partial \Gamma}{\partial n}-u \frac{\partial G}{\partial n}\right) d \sigma+\iint_{D} \Gamma \Delta^{2} u d A .
$$


This identity may now be applied to problem $(1) /\left(b^{\prime}\right)$ to yield the solution of this nonhomogeneous boundary value problem in the form

$$
u(P)=\iint_{\partial D}\left(g_{2}(Q) \frac{\partial \Gamma(Q, P)}{\partial n}-g_{1}(Q) \frac{\partial G(Q, P)}{\partial n}\right) d \sigma+\iint_{D} F(Q) \Gamma(Q, P) d A
$$

Thus for any domain $D$ for which the Green's functions $\Gamma$ and $G$ and their normal derivatives exist and are regular, the functions $g_{1}$ and $g_{2}$ are continuous on $\partial D$, and $F$ is continuous on $D$, the solution of boundary value problem $(1) /\left(b^{\prime}\right)$ can be represented by (9).

The fact that the solution of problem $(1) /\left(b^{\prime}\right)$ is unique can be demonstrated directly and without the use of Green's identities. For assume there exist two solutions $u_{1}$ and $u_{2}$ of problem $(1) /\left(b^{\prime}\right)$ and $\mid$ let $u=u_{i}-u_{2}$. Then $u$ satisfies the homogeneous boundary value problem $\Delta^{2} u=0$ in $D ; u=0=\Delta u$ on $\partial D$. Now let $v=\Delta u$. Then this function satisfies the Dirichlet problem $\Delta v=0$ in $D ; v=0$ on $\partial D$, which is known to have the unique solution $v=0$ in $D$. Hence the original problem also reduces to the Dirichlet problem $\Delta u=0$ in $D ; u=0$ on $\partial D$. Since this problem has the unique solution $u \equiv 0$ in $D$, it follows that $u_{1}=u_{2}$ throughout $D$.

\section{Properties of the supported plate Green's function}

The supported plate Green's function has a physical interpretation similar to that of the corresponding clamped plate Green's function. For let a single point force of the $\delta$-function type be applied normal to the plate at $P$, then the deflection of the plate at $Q$ is given by $\Delta^{2} u=\delta(Q$ $-P$ ) in $D ; u=0=\Delta u$ on $\partial D$. The solution of this problem by (9) is

$$
u(P)=\iint_{D} \delta(Q-P) \Gamma(Q, P) d A=\Gamma(Q, P) .
$$

Hence $\Gamma(Q, P)$ represents the deflection at $Q$ resulting from a unit normal force applied at $P$ to a plane thin elastic plate which occupies the domain $D$ and is supported along its edge $\partial D$.

A number of other properties of $\Gamma(Q, P)$ follow immediately from the easily demonstrated composition formula [7]

$$
\Gamma(Q, P)=\iint_{D} G(Q, R) G(R, P) d A
$$

where $R \in D$ and $d A$ here represents the area element in the variables used to denote the point $R$. Since the harmonic Green's function $G(Q, P)$ is known to be symmetric and to have the same sign throughout $D$, it follows from (10) that these properties also hold for $\Gamma(Q, P)$ in $D$. The latter property is noteworthy, since it is known that there exist domains for which the clamped plate Green's function changes sign [6]. Interpreted physically, the symmetry of $\Gamma(Q, P)$ asserts a version of Maxwell's reciprocity principle, namely that the deflection at a point $Q$ due to a unit force applied at the point $P$ equals the deflection at $P$ due to the same force applied at $Q$.

The composition formula (10) may also be used to derive an upper bound for $\Gamma(Q, P)$ on a bounded domain $D$. For, using the maximum principle for harmonic functions, it is easily shown [12] that the harmonic Green's function is bounded by $0<G(Q, P) \leq(1 / 2 \pi) \log (h / r)$ on $D$, where $h$ is the diameter of $\bar{D}$. Now, letting the closed domain $\bar{D}$ lie entirely in the interior 
of a disk with radius $h$ and center at an arbitrary point of $D$, it follows that

$$
0<\iint_{D} G^{2}(Q, P) d A \leq \frac{1}{4 \pi^{2}} \int_{0}^{2 \pi} \int_{0}^{h} \log ^{2} \frac{h}{r} r d r d \vartheta=\frac{h^{2}}{8 \pi} .
$$

Applying the Cauchy-Schwarz inequality to (10) and using (11) then yields

$$
|\Gamma(Q, P)|^{2}=\left|\iint_{D} G(Q, R) G(R, P) d A\right|^{2} \leq \iint_{D} G^{2}(Q, R) d A \cdot \iint_{D} G^{2}(R ; P)^{2} d A s\left(\frac{5 h_{1}^{2}}{8 \pi}\right)^{2},
$$

or $0<|\Gamma(Q, P)| \leq h^{2} / 8 \pi$ throughout $D$.

\section{The supported plate boundary vahue problem for the disk}

The supported plate Green's function for the disk can be computed by a complex variable method first suggested for the clamped plate by Garabedian $[5,6]$. Let the disk in the complex $z$-plane be $D=\{z|| z \mid<R\}$, let the points $Q$ and $P$ in the Green's function $\Gamma(Q, P)$ be denoted by $z=s \mathrm{e}^{\mathrm{i} \theta} \in \bar{D}$ and $\zeta=\rho \mathrm{e}^{\mathrm{i} \varphi} \in D$, respectively, and let $t=|z-\zeta|$. In accordance with (6) let the desired Green's function be written in the form

$$
\Gamma(z, \zeta)=\frac{1}{8 \pi}|z-\zeta|^{2} \log |z-\zeta|+\operatorname{Re}\left\{\left(z \bar{z}-R^{2}\right) \oplus(z)+\Psi(z)\right\},
$$

where the first term on the right is the fundamental solution $S(r)$ and the second term is an arbitrary biharmonic function represented by the appropriate form of Goursat's formula (3) in terms of two harmonic functions. The harmonic functions $\operatorname{Re} \Phi(z)$ and $\operatorname{Re} \Psi(z)$ are now determined from the two boundary conditions of the supported plate Green's function, i.e. $\Gamma=0$ and $\Delta \Gamma=0$ on $\partial D$. The application of these boundary conditions is facilitated by introducing the inverse point $\zeta$ with respect to the circle $|z|=R$, namely $R^{2} / \bar{\zeta}$. The relation $R|z-\zeta|=\mid R^{2}-$ $\bar{\zeta} z \mid$ then holds on $\partial D$, and applying the first boundary condition, $\Gamma=0$ on $\partial D$, yields

$$
\operatorname{Re} \Psi(z)=-\frac{1}{8 \pi}|z-\zeta|^{2} \log \frac{\left|R^{2}-\bar{\zeta} z\right|}{R}
$$

on $\partial D$, and hence

$$
\Gamma(z, \zeta)=\frac{1}{8 \pi}|z-\zeta|^{2} \log \left|\frac{R(z-\zeta)}{R^{2}-\bar{\zeta} z}\right|+\left(z \bar{z}-R^{2}\right) \operatorname{Re} \Phi(z)
$$

for all $z \in \bar{D}$. The Laplacian of this function is most easily computed by applying this operator in its complex form, and is

$$
\Delta \Gamma=4 \frac{\partial^{2} \Gamma}{\partial z \partial \bar{z}}=\frac{1}{2 \pi} \log \left|\frac{R(z-\zeta)}{R^{2}-\bar{\zeta} z}\right|+\frac{1}{2 \pi} \operatorname{Re}\left\{\frac{R^{2}-|\zeta|^{2}}{R^{2}-\bar{\zeta} z}\right\}+4 \operatorname{Re}\left\{\frac{\partial z \Phi(z)}{\partial z}+\left(z \bar{z}-R^{2}\right) \Delta \Phi\right\} .
$$

Applying the second boundary condition, $\Delta \Gamma=0$ on $\partial D$, then yields

$$
\operatorname{Re}\left\{\frac{\partial z \Phi(z)}{\partial z}+\frac{1}{8 \pi} \frac{R^{2}-|\zeta|^{2}}{R^{2}-\bar{\zeta} z}\right\}=0
$$


on $\partial D$, or by analytic continuation,

$$
\Phi(z)=\frac{1}{8 \pi} \frac{R^{2}-|\zeta|^{2}}{\bar{\zeta} z} \log \frac{R^{2}-\bar{\zeta} z}{R^{2}}
$$

for all $z \in \bar{D}$. Thus, the supported plate Green's function for the disk in complex form is

$$
\Gamma(z, \zeta)=\frac{1}{8 \pi}|z-\zeta|^{2} \log \left|\frac{R(z-\zeta)}{\mid R^{2}-\bar{\zeta} z}\right|-\frac{1}{8 \pi}\left(R^{2}-|z|^{2}\right)\left(R^{2}-|\zeta|^{2}\right) \operatorname{Re}\left\{\frac{\log \left[\left(R^{2}-\bar{\zeta} z\right) / R^{2}\right]}{\bar{\zeta} z}\right\} .
$$

On introducing the distances $r=|z-\zeta|$ and $\tilde{r}=\left|z-R^{2} / \bar{\zeta}\right|$ and making use of

$$
\bar{\zeta}_{z}=\rho s(\cos (\vartheta-\varphi)+\mathrm{i} \sin (\vartheta-\varphi)) \text { and } \log \frac{R^{2}-\bar{\zeta} z}{R^{2}}=\log \frac{\rho \tilde{r}}{R^{2}}+\mathrm{i} \tan ^{-1} \frac{\rho s \sin (\vartheta-\varphi)}{\rho s \cos (\vartheta-\varphi)-R^{2}}
$$

this Green's function may be written in the real form

$$
\begin{aligned}
\Gamma(Q, P)= & \frac{1}{8 \pi} r^{2} \log \frac{R r}{\rho \tilde{r}}-\frac{1}{8 \pi} \frac{\left(R^{2}-\rho^{2}\right)\left(R^{2}-s^{2}\right)}{\rho s} \\
& \times\left[\cos (\vartheta-\varphi) \log \frac{\rho \tilde{r}}{R^{2}}+\sin (\vartheta-\varphi) \tan ^{-1} \frac{\rho s \sin (\vartheta-\varphi)}{\rho s \cos (\vartheta-\varphi)-R^{2}}\right],
\end{aligned}
$$

where $Q(s, \vartheta)$ and $P(\rho, \varphi)$ are these points in polar coordinates, $r$ is the distance between them, and $\tilde{r}$ is the distance from $Q$ to the inverse point of $P$ in the circle $s=R$. On using the identity $\rho^{2} \tilde{r}^{2}-R^{2} r^{2}=\left(R^{2}-\rho^{2}\right)\left(R^{2}-s^{2}\right)$ the quantity $\tilde{r}$ can be eliminated and (12) becomes

$$
\begin{aligned}
\Gamma(Q, P)= & -\frac{1}{16 \pi} r^{2} \log \left[1+\frac{\left(R^{2}-\rho^{2}\right)\left(R^{2}-s^{2}\right)}{R^{2} r^{2}}\right] \\
& -\frac{1}{16 \pi} \frac{\left(R^{2}-\rho^{2}\right)\left(R^{2}-s^{2}\right)}{\rho s}\left\{\cos (\vartheta-\varphi) \cdot \log \left(r^{2}+\frac{\left(R^{2}-\rho^{2}\right)\left(R^{2}-s^{2}\right)}{R^{2}}\right)\right. \\
& \left.+2 \sin (\vartheta-\varphi) \tan ^{-1} \frac{\rho s \sin (\vartheta-\varphi)}{\rho s \cos (\vartheta-\varphi)-R^{2}}\right\} .
\end{aligned}
$$

The symmetry of this Green's function is now evident.

By letting the variable point $Q$ approach the "load point" $P$, so that $s=\rho, \theta=\varphi$, and $r=0$, it is easily seen from (13) that the deflection of the supported circular plate under a unit load at $P$ is

$$
\Gamma(P, P)=-\frac{1}{8 \pi} \frac{\left(R^{2}-Q^{2}\right)^{2}}{P^{2}} \log \frac{R^{2}-Q^{2}}{R} .
$$

Figure 1a exhibits a graph of the supported plate Green's function (13) with the unit force applied upwards to the horizontally held disk at a point $3 / 10$ of the distance along its diameter. The figure thus shows the bending of a thin elastic circular plate which is simply supported along its edge and subjected to this point force. (For illustrative purposes the scale of the deflection has been magnified.) For comparison, a graph of the clamped plate Green's function for the disk subject to an identically located unit point force is shown in Figure 1b. The clamped plate Green's function $\Lambda(Q, P)=S(r)+\lambda(Q, P)$ is defined in the same way as that of the supported plate; however, the second boundary condition in the definition is replaced by $\partial \lambda / \partial n$ 


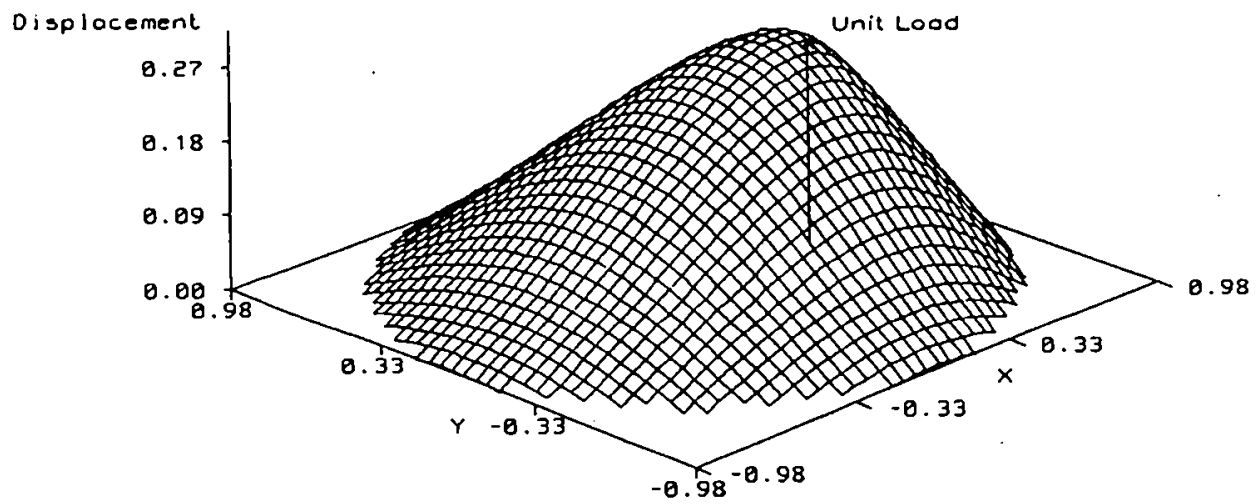

Fig. 1a: Supported plate unit disk

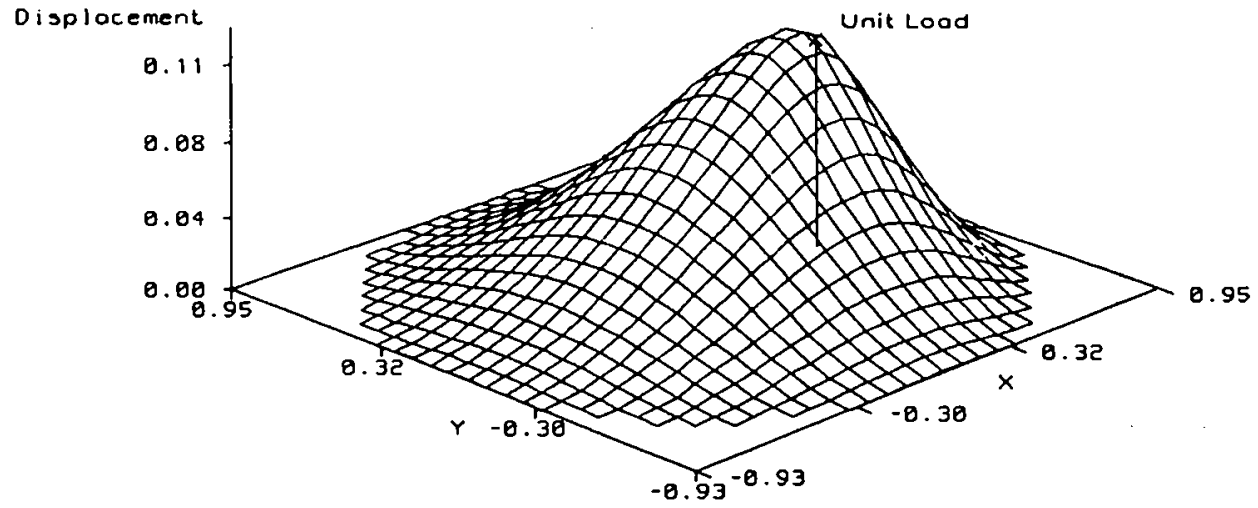

Fig. 1b: Clamped plate unit disk

$=-\partial S / \partial n$ on $\partial D$. For the disk it may be shown to be $[6,11]$

$$
\Lambda(Q, P)=-\frac{1}{16 \pi} r^{2} \log \left[1+\frac{\left(R^{2}-\rho^{2}\right)\left(R^{2}-s^{2}\right)}{R^{2} r^{2}}\right]+\frac{1}{16 \pi} \frac{\left(R^{2}-\rho^{2}\right)\left(R^{2}-s^{2}\right)}{R^{2}},
$$

.where the same notation as in (13) has been used. The graphs in Figure 1, as well as those in Figure 2 in the next section, were computed and plotted using an SAS/GRAPH computer graphics package by Mr. James $\mathrm{M}$. Thompson as part of his master's thesis project at The American University. 
It may be observed that the graphs of these Green's functions are very similar. However, it is evident from the graph of the clamped plate that the normal derivative of the deflection vanishes at its edge, whereas for the supported plate it does not. In both graphs the maximum deflection does not take place at the (asymmetrically placed) load point, but rather at a nearby point. This is due to the fact that a plate, unlike a membrane, resists bending.

To obtain the solution of the supported plate boundary value problem for the disk, the normal derivatives of $\Gamma(Q, P)$ and $G(Q, P)$ on $\partial D$ must now be computed and substituted into (9). Making use of the relations

$$
r^{2}=s^{2}+\rho^{2}-2 \rho s \cdot \cos (\vartheta-\varphi) \text { and } \tilde{r}^{2}=s^{2}+R 4 / \rho^{2}-\left(2 R^{2} s / \rho\right) \cdot \cos (\vartheta-\varphi)
$$

and of $s=R$ and $\rho \tilde{r}=R r$ on $\partial D$, the normal derivative of $\Gamma(Q, P)$ on $\partial D$ may be obtained from (12) to be

$$
\left(\frac{\partial \Gamma}{\partial n}\right)_{\partial D}=\frac{1}{8 \pi} \frac{\left(R^{2}-\rho^{2}\right)}{R \rho}\left[\rho+2 R \cos (\vartheta-\varphi) \cdot \log \frac{r}{R}+2 \sin (\vartheta-\varphi) \cdot \tan ^{-1} \frac{\rho \sin (\vartheta-\varphi)}{\rho \cos (\vartheta-\varphi)-R}\right] .
$$

The harmonic Green's function for the disk is $G(Q, P)=(1 / 2 \pi) \log (\rho \tilde{r} / R r)$, and its normal derivative on $\partial D$ is the negative of the well-known Poisson kernel

$$
\left(\frac{\partial G}{\partial n}\right)_{\partial D}=-\frac{1}{2 \pi} \frac{R^{2}-\rho^{2}}{R r^{2}}
$$

Substituting (14) and (15) into (9) with $F(Q)=0$ then yields the solution of the homogeneous supported plate boundary value problem $(2) /\left(b^{\prime}\right)$ for the disk,

$$
\begin{aligned}
u(\rho, \varphi)= & \frac{R^{2}-\rho^{2}}{2 \pi} \int_{0}^{2 \pi} g_{1}(\vartheta) r^{-2} d \vartheta+\frac{R^{2}-\rho^{2}}{8 \pi \rho} \int_{0}^{2 \pi} g_{2}(\vartheta)\left[\rho+2 R \cos (\vartheta-\varphi) \cdot \log \frac{r}{R}\right. \\
& \left.+2 R \sin (\vartheta-\varphi) \cdot \tan ^{-1} \frac{\rho \sin (\vartheta-\varphi)}{\rho \cos (\vartheta-\varphi)-R}\right] d \vartheta .
\end{aligned}
$$

\section{The supported plate boundary value problem for the half-plane}

The supported plate Green's function for the half-plane may be obtained by a similar method to that used for the disk in the last section. For definiteness consider the right half-plane $D=$ $\{z \mid \operatorname{Re} z>0\}$ and let the points $P$ and $Q$ be denoted by $z=x+\mathrm{i} y \in \bar{D}$ and $\zeta=\xi+i \eta \in \mathrm{D}$, respectively. The appropriate form of the Green's function for $D$ is

$$
\Gamma(z, \zeta)=\frac{1}{8 \pi}|z-\zeta|^{2} \log |z-\zeta|+\operatorname{Re}\{(z+\bar{z}) \varphi(z)+\psi(z)\},
$$

where the harmonic functions $\operatorname{Re} \varphi(z)$ and $\operatorname{Re} \psi(z)$ are again determined from the two boundary conditions of $\Gamma(z, \zeta)$ on $\partial D$. Let the image point $-\bar{\zeta}$ of $\zeta$ in $\partial D$ be introduced so that $|z-\zeta|=$ $|z+\bar{\zeta}|$ on $\partial D$. Applying the first boundary condition, $\Gamma=0$ on $\partial D$, to (17) immediately yields

$$
\operatorname{Re} \psi(z)=-\frac{1}{8 \pi}|z-\zeta|^{2} \log |z+\bar{\zeta}|
$$

on $\partial D$, and hence 


$$
\Gamma(z, \zeta)=\frac{1}{8} \frac{1}{\pi}|z-\zeta|^{2} \log \left|\frac{z-\zeta}{z+\bar{\zeta}}\right|+(z+\bar{z}) \operatorname{Re} \varphi(z)
$$

for all $z \in \bar{D}$. Applying the second boundary condition, $\Delta \Gamma=0$ on $\partial D$, to (18) leads to

$$
\operatorname{Re}\left\{\varphi^{\prime}(z)+\frac{1}{8} \frac{\zeta+\bar{\zeta}}{z+\bar{\zeta}}\right\}=0
$$

on $\partial D$. Thus, the supported plate Green's function for the right half-plane becomes

$$
\Gamma(z, \zeta)=\frac{1}{8 \pi}|z-\zeta|^{2} \log \left|\frac{z-\zeta}{z+\bar{\zeta}}\right|-\frac{1}{8 \pi}(z+\bar{z})(\zeta+\bar{\zeta}) \log |z+\bar{\zeta}| .
$$

On using the identity $(z+\bar{z})(\zeta+\bar{\zeta})=|z+\bar{\zeta}|^{2}-|z-\zeta|^{2}$ this reduces to the remarkably simple form

$$
\Gamma(z, \zeta)=\frac{1}{8 \pi}|z-\zeta|^{2} \log |z-\zeta|-\frac{1}{8 \pi}|z+\bar{\zeta}|^{2} \log |z+\bar{\zeta}| .
$$

If the distances $r=|z-\zeta|$ and $\widetilde{r}=|z+\bar{\zeta}|$ are introduced this may be written in the real form

$$
\Gamma(Q, P)=\frac{1}{8 \pi}\left(r^{2} \log r-\widetilde{r}^{2} \log \tilde{r}\right) .
$$

The distances $r$ and $\widetilde{r}$ may in fact be interpreted in more general terms as the distance from $Q$ to $P$ and the distance from $Q$ to the image point $\widetilde{P}$ of $P$ in any straight line boundary $\partial D$, respectively. Interpreted in this way, relation (19) represents the supported plate Green's function for any half-plane. According to the remark made in Section 3, physically this Green's function represents the deflection of the plate at any point $Q$ due to two individual point loads, each acting perpendicularly to the plate but in opposite directions at $P$ and $\widetilde{P}$. On taking the negative Laplacian of (19), this expression reduces, in agreement with (8), to the harmonic Green's function for the half-plane, namely $G(Q, P)=(1 / 2 \pi) \log (\widetilde{r} / r)$.

A graph of the supported plate Green's function (19) is shown in Figure 2a. For comparison, the clamped plate Green's function for the half-plane $\Lambda(Q, P)=(1 / 16 \pi)\left[r^{2} \log \left(r^{2} / \gamma^{2}\right)-\left(r^{2}\right.\right.$ $\left.-\widetilde{r}^{2}\right]$ under the same point load is plotted in Figure $2 \mathrm{~b}$. Again, these graphs representing the

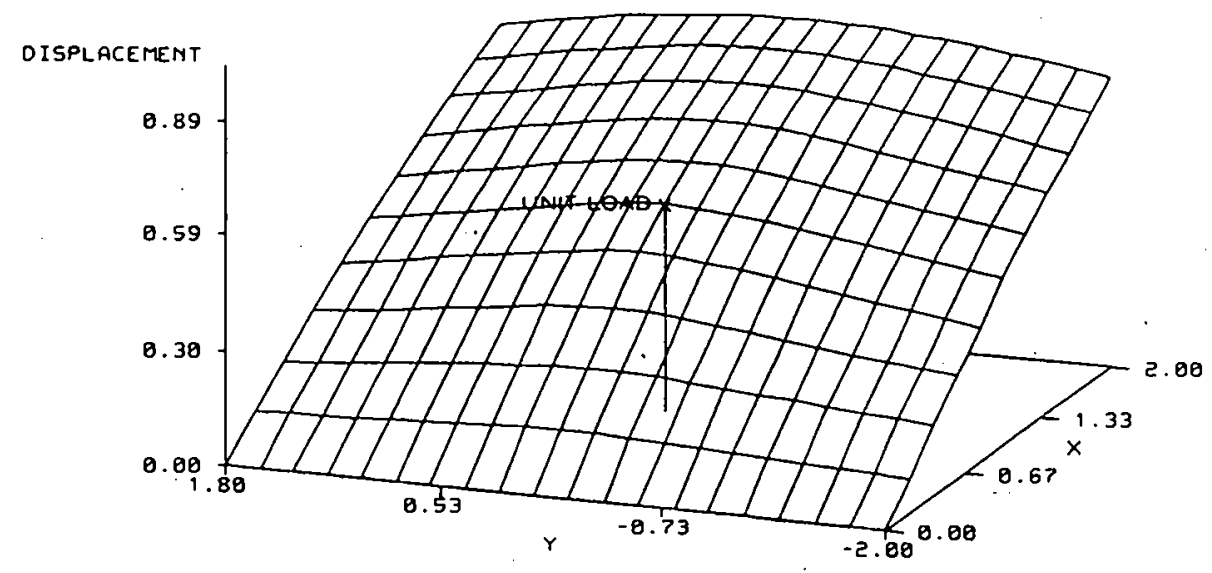

Fig. 2a: Supported plate right half-plane 


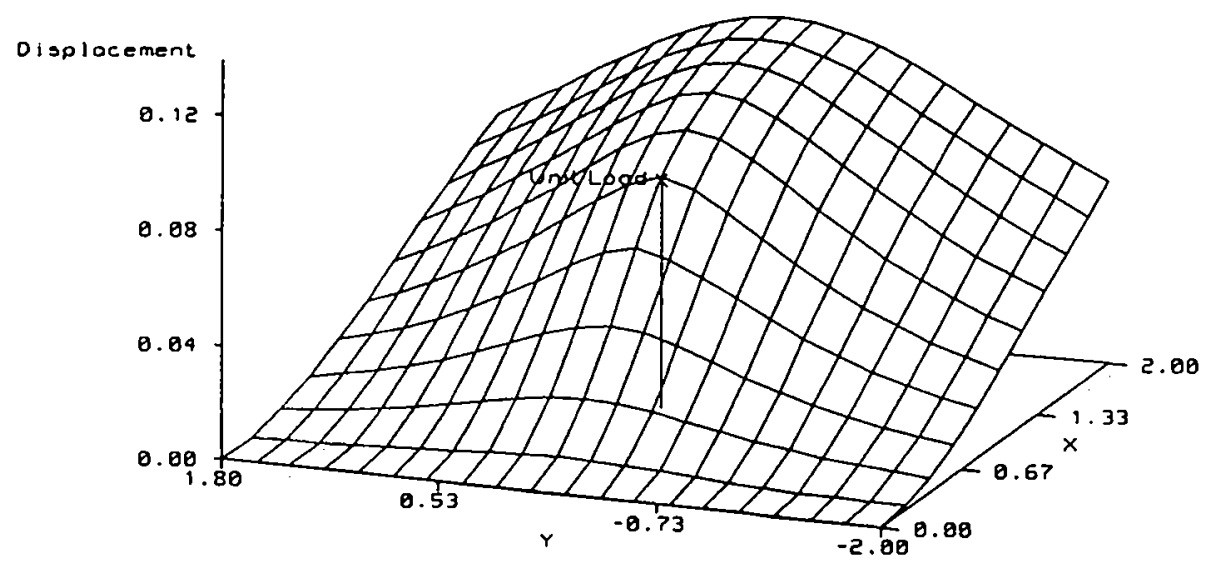

Fig. 2b: Clampod plate right half-plane

deflection of the two plates are quite similar, except that the normal derivative of the deflection vanishes at the straight edge of the clamped plate but not at that of the supported plate. Moreover, as for the disk, the maximum deflection for the half-plane does not occur at the load point.

When the normal derivatives of $\Gamma(Q, P)$ and $G(Q, P)$ for the right half-plane are computed on its boundary $\partial D$ and substituted into $(9)$ with $F(Q)=0$, the solution of the homogeneous supported plate boundary value problem $(2) /\left(b^{\prime}\right)$ for the right half-plane is obtained, namely

$$
u(\xi, \eta)=\frac{\xi}{\pi} \int_{-\infty}^{+\infty} g_{1}(y) \frac{1}{\xi^{2}+(y-\eta)^{2}} d y+\frac{\xi}{4 \pi} \int_{-\infty}^{+\infty} g_{2}(y) \log \left[\xi^{2}+(y-\eta)^{2}\right] d y .
$$

\section{Direct method for the supported plate boundary value problem}

The direct method developed by Almansi [1] for the clamped plate may be extended to solve the supported plate boundary value problems for the disk and the half-plane. This will serve as a check on the solutions obtained by the Green's function method in the last two sections.

To solve the homogeneous supported plate boundary value problem $(2) /\left(b^{\prime}\right)$ for the disk in polar coordinates $D=\{(\rho, \varphi) \mid 0 \leq \rho \leq R, 0 \leq \varphi \leq 2 \pi\}$ let a Goursat solution (3) be chosen in the form

$$
u(\rho, \varphi)=\left(\rho^{2}-R^{2}\right) \oplus(\rho, \varphi)+\Psi(\rho, \varphi)
$$

where $\Phi$ and $\Psi$ are harmonic functions in $\rho<R$. The first boundary condition, $u=g_{1}$ on $\partial D=$ $\{(\rho, \vartheta) \mid \rho=R, 0 \leq \Im \leq 2 \pi\}$, when applied to $(21)$ yields $u(R, \vartheta)=\Psi(R, \vartheta)=g_{1}(\vartheta)$ on $\partial D$, so that $\Psi$ satisfies the Dirichlet problem 
$\Delta \Psi=0$ in $D, \Psi=g_{1}(\vartheta)$ on $\partial D$.

The solution of this Dirichlet problem is given by Poisson's integral

$$
\Psi(\rho, \varphi)=\frac{1}{2 \pi} \int_{0}^{2 \pi} g_{1}(\vartheta) \frac{R^{2}-Q^{2}}{r^{2}} d \vartheta,
$$

where $r^{2}=R^{2}+\rho^{2}-2 \rho R \cos (\vartheta-\varphi)$. The second boundary condition, $\Delta u=g_{2}$ on $\partial D$, when applied to (21) yields

$$
\left.(\Delta u)\right|_{\partial D}=\left.4[\partial(\rho \Phi) / \partial \rho]\right|_{\rho=R}=g_{2}(\vartheta)
$$

on $\partial D$, so that the harmonic function $\partial(\rho \Phi) / \partial \rho$ satisfies the Dirichlet problem

$$
\Delta[\partial(\rho \Phi) / \partial \rho]=0 \text { in } D,\left.\quad[\partial(\rho \Phi) / \partial \rho]\right|_{\rho=R}=4^{-1} g_{2}(\vartheta) \text { on } \partial D \text {. }
$$

The solution of this Dirichlet problem is $\partial(\rho \Phi) / \partial p=1 / 8 \pi \int_{0}^{2 \pi} g_{2}(\theta)\left(\left(R^{2}-p^{2}\right) / r^{2}\right) d \vartheta$, so that

$$
\Phi(\rho, \varphi)=\frac{1}{8 \pi} \int_{0}^{2 \pi} g_{2}(\vartheta) \int_{0}^{\rho} \frac{R^{2}-\rho^{2}}{r^{2}} d \rho d \vartheta .
$$

Substituting (22) and (23) in (21) the solution of the homogeneous supported plate boundary value problem for the disk takes the compact form

$$
u(\rho, \varphi)=\frac{1}{2 \pi} \int_{0}^{2 \pi} g_{1}(\vartheta) \frac{R^{2}-\rho^{2}}{r^{2}} d \vartheta-\frac{R^{2}-\rho^{2}}{8 \pi \rho} \int_{0}^{2 \pi} g_{2}(\vartheta) \int_{0}^{\rho} \frac{R^{2}-\rho^{2}}{r^{2}} d \rho d \vartheta .
$$

The inner integral in (24) can be evaluated by elementary integration, and becomes

$$
\int_{0}^{\rho} \frac{\rho^{2}-R^{2}}{r^{2}} d \rho=\rho+2 R \cos (\vartheta-\varphi) \cdot \log \frac{r}{R}-2 R \sin (\vartheta-\varphi) \cdot \tan ^{-1} \frac{\rho \sin (\vartheta-\varphi)}{R-\rho \cos (\theta-\varphi)} .
$$

When this integral is substituted back into (24) the resulting solution reduces to (16).

Problem (2)/(b') for the right half-plane $D=\{(\xi, \eta) \mid \xi>0\}$ may be solved in a similar manner by the direct method. This time the appropriate form of the Goursat solution (3) is

$$
u(\xi, \eta)=\xi \varphi(\xi, \eta)+\psi(\xi, \eta),
$$

where $\varphi$ and $\psi$ are harmonic functions on $D$. The boundary conditions $u=g_{1}$ and $\Delta u=g_{2}$ on $\partial D$ $=\{(\xi, \eta) \mid \xi=0\}$ in problem $(2) /\left(b^{\prime}\right)$ for the right half-plane lead to the boundary values

$$
\psi(0, \eta)=g_{1}(\eta),\left.\quad(\Delta u)\right|_{\partial D}=2 \frac{\partial \varphi}{\partial \xi}(0, \eta)=g_{2}(\eta)
$$

for these functions. The corresponding Dirichlet problems for the harmonic functions $\psi$ and $\partial \varphi / \partial \xi$ are solved by the applicable Poisson integrals for the half-plane, namely

$$
\psi(\xi, \eta)=\frac{\xi}{\pi} \int_{-\infty}^{+\infty} g_{1}(y) \frac{1}{\xi^{2}+(y-\eta)^{2}} d y \text { and } \frac{\partial \varphi}{\partial \xi}(\xi, \eta)=\frac{\xi}{2 \pi} \int_{-\infty}^{+\infty} g_{2}(y) \frac{1}{\xi^{2}+(y-\eta)^{2}} d y,
$$


respectively. Substituting $\varphi(\xi, \eta)$ and $\psi(\xi, \eta)$ from (26) back into (25) yields

$$
u(\xi, \eta)=\frac{\xi}{\pi} \int_{-\infty}^{+\infty} g_{1}(y) \frac{1}{\xi^{2}+(y-\eta)^{2}} d y+\frac{\xi}{2 \pi} \int_{-\infty}^{+\infty} g_{2}(y) \int_{0}^{\xi} \frac{\xi}{\xi^{2}+(y-\eta)^{2}} d \xi d y,
$$

which agrees with (20).

\section{REFERENCES}

[1] ALMANSI, E.: Sull integraziono dell equazione differenziale $\Delta^{2} \Delta^{2}=0$. Atti Reale Accad. Sci. Torino $31(1895 / 96), 881-888$.

[2] BERGMAN, S. and M. SCHIFFER: Kernel Functions and Elliptic Partial Differential Equations in Mathematical Physics. New York: Acad. Press 1953.

[3] DEAN, W. R.: The Green's function of an elastic plato. Proc. Cambr. Phil. Soc. 49 (1953), $319-326$

[4] FRANK, P. and R. VON MISES: Die Differential- und Intogralgleichungen der Mechanik und Physik. 2 Vols., 2nd ed. Braunschweig: Vieweg 1930 and 1935; Reprint: Now York: Dover 1961.

[5] GARABEDIAN, P.R.: Applications of analytic continuation to the solution of boundary value problems. J. Rat. Mech. Anal. 3 (1954), 383 - 393.

[6] Garabedian, P.R.: Partial Differential Equations. Now York: Wiley 1964.

[7] GoUld, S.H.: Variational Methods for Eigenvalue Problems. Toronto: University of Toronto Press 1957.

[8] GourSAT, E.: Sur I' equation $\Delta \Delta u=0$. Bull. Soc. Math. Franco 26 (1898), $236-237$.

[9] HADAMARD, J.: Mémoire sur le probleme d'analyse relatif à l'óquilibre des plaques élastique encastróes. Mémoires presentés par divers savants à lAcad. Sci. (2) 3 (1908), $1-128$.

[10] LAURICELLA, G.: Integrazione dell equazione $\Delta^{2}\left(\Delta^{2} u\right)=0$ in un campo di forma circolare. Atti Reale Accad. Sci. Torino 31 (1895/96), 1010 - 1018.

[11] LoVE, A. E. H.: A Treatise on the Mathematical Theory of Elasticity. 4 th ed. Cambridge: Cambridge Univ. Press 1927. Reprint: New York: Dover 1944.

[12] PICARD. E.: Leçons sur quelques problomes aux limites do la théorie des équations differentielles. Paris: Gauthier-Villars 1930.

[13] REISSNER, E.: Uber die Biegung dor Kreisplatte mit exzentrischor Einzellast. Math. Ann. 11 (1935), $777-780$.

[14] VOLTERRA, V.: Osservazioni sulla nota procedento del Prof. Lauricella o sopra una nota di analogo dell'Ing. Almansi. Atti Reale Accad. Sci. Torino 31 (1895/96), 1018 - 1021.

[15] WEINSTOCK, R.: Calculus of Variations. New York: McGraw-Hill 1952.

Received 18.02.1992

Prof. Dr. Stoven H. Schot

The American University

Department of Mathematics

Weshington, DC 20016, USA 